\title{
MANIFESTACIÓN DE HOMOFOBIA INTERNALIZADA EN ADOLESCENTES Y JÓVENES AL NORTE DE MÉXICO
}

\section{MANIFESTATION OF INTERNALIZED HOMOPHOBIA IN ADOLESCENTS AND YOUNG ADULTS AT THE NORTH OF MEXICO}

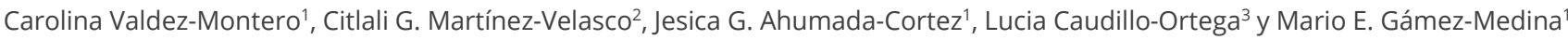 \\ ${ }^{1}$ Universidad Autónoma de Sinaloa, México \\ universidad Juárez Autónoma de Tabasco, México \\ 3Universidad de Guanajuato, México
}

\section{Abstract}

Internalized homophobia has been linked to mental health problems such as stress, anxiety, depression and suicidal ideation. The aim of this study was to determine the association between sex, age and internalized homophobia in adolescents and youth. It was a crosssectional descriptive-correlational study. It was conducted in 246 adolescents and youth from two public institutions and the Internalized Homonegativity Scale (HNI-16) was used. The results indicate that men have a higher level of internalized homophobia compared to women $(U=5,077.00, p<.001)$ and age is negatively related to internalized homophobia $(r=-.22, p<.001)$. The model was significant showing that $11.2 \%$ of the variation in internalized homophobia was due to sex and age $\left(F_{[2,243]}=15.40, p<.001\right)$.

Keywords: Depression, discrimination, homophobia, homosexuality.

\section{Resumen}

La homofobia internalizada se ha relacionado con problemas de salud mental tales como estrés, ansiedad, depresión e ideaciones suicidas. El objetivo del presente estudio fue determinar la asociación entre el sexo, la edad y la homofobia internalizada en adolescentes y jóvenes. Se realizó un estudio descriptivo correlacional de corte transversal en 246 adolescentes y jóvenes de dos instituciones públicas y se utilizó la Escala de Homonegatividad Internalizada (HNI-16). Los resultados indican que los hombres presentan mayor nivel de homofobia internalizada en comparación a las mujeres $(U=5,077.00, p<.001)$ y la edad se relaciona negativamente con la homofobia internalizada $(r=-.22, p<$ .001). El modelo fue significativo al demostrar que el $11.2 \%$ de la variación en la homofobia internalizada se debió al sexo y la edad $\left(F_{[2,243]}=15.40, p<.001\right)$.

Palabras clave: Depresión, discriminación, homofobia, homosexualidad. 
La orientación sexual de personas no heterosexuales suele generar creencias negativas y prejuicios homofóbicos en la sociedad, dado que se les asocia con comportamientos que van en contra de las normas culturales establecidas, así como con enfermedades como el VIH, las ITS, entre otras. Algunos autores señalan que las personas con una identidad y orientación no heterosexual pueden desarrollar actitudes negativas en ellos mismos y hacia los otros con la misma orientación, debido a discriminación social y el rechazo de la propia orientación sexual, esto puede ser transformado en miedo y rechazo a la aceptación de la propia diversidad sexual (Fernández \& Vázquez, 2013; Lozano \& Díaz-Loving, 2010; Moral \& Valle, 2013).

Estos prejuicios sociales traen consigo rechazo y exclusión social que suelen producir efectos negativos en la salud mental de las personas, entre estos se encuentra un mayor consumo de drogas lícitas e ilícitas, pensamiento suicida, bullying, rechazo familiar, síntomas depresivos, trastorno de ansiedad generalizada, entre otros. Aunque las actitudes sociales hacia la homosexualidad han cambiado a través del tiempo aún se continúan manteniendo los valores tradicionales que enfatizan la preservación del apellido familiar, las relaciones entre hombre y mujer y rechazan totalmente la unión entre las personas del mismo sexo, esto genera sentimientos de culpa y vergüenza.

La homofobia se define no propiamente como una fobia, sino como un rechazo y desprecio dirigida a las personas no heterosexuales (Barrientos \& Cárdenas, 2013). Las expresiones de la misma varían en diferentes espacios físicos, geográficos e históricos, de tal forma que se trata de una construcción social y cultural (Lozano \& Rocha, 2011). Las personas homosexuales internalizan la culpa, vergüenza, discriminación y prejuicios generando la homofobia internalizada, que es definida como el rechazo a su orientación homosexual, que implica conflictos internos entre lo que la persona quiere ser y lo que debe ser para la sociedad en relación a la experimentación de su sexualidad (Herek, 2004).

Estudios han reportado que mayores niveles de homofobia internalizada se asoció con problemas de salud mental principalmente con manifestaciones de síntomas depresivos y ansiedad (Newcomb \& Mustanski, 2010). Asimismo, Xu, Zheng, Xu, \& Zheng (2017) encontraron en su estudio que las personas que experimentaron mayores niveles de homofobia internalizada tenían mayor probabilidad de presentar mayores niveles de estrés. Por lo cual se puede señalar que los eventos de auto rechazo y discriminación incrementan el riesgo de desarrollar un trastorno mental.

Es preciso señalar que las personas en la etapa de la adolescencia y la juventud son más vulnerables, dado que surgen cambios físicos, psicológicos y emocionales, por la transición de la niñez a la edad adulta; los adolescentes tienen que asumir una nueva imagen de sí mismos (as), explorar y reafirmar su sexualidad (Caricote, 2009). En la etapa de la adolescencia y la juventud se experimentan diversos cambios en las esferas biopsicosociales donde se busca tener acceso a nuevas sensaciones que le permitan al joven experimentar diversos comportamientos que pueden ser de riesgo para su salud, como el consumo de alcohol y tabaco, tener relaciones sexuales de riesgo que incrementan y proporcionan nuevas emociones (Silva-Escorcia \& MejíaPérez, 2015).

Los adolescentes y jóvenes pueden presentar conflictos internos respecto al reconocimiento y aceptación de su identidad sexual, principalmente quienes viven en contextos económicos desfavorables y culturas heteronormativas. El conflicto con la identidad sexual tiene fuerte una relación con las creencias sociales, prejuicios y estigmas en relación con la homosexualidad (homofobia), que genera una gran angustia entre los adolescentes y jóvenes quienes se encuentran en el proceso de definición y aceptación de su identidad sexual (Kaysen et al., 2014; Peixoto, Fonseca, Almeida, \& Almeida, 2012; Teixeira-Filho, Rondini, \& Bessa, 2011). El adolescente o joven que vive bajo rechazo social, tiende a sentirse solo, sin apoyo y esto puede disminuir su comunicación e interacción social, se siente culpable, rechaza su propia identidad tiende a aislarse y a evitar acudir a los profesionales de la salud, así incrementa su riesgo de conductas de riesgo, debido a sus pobres mecanismos de afrontamiento personales y sociales.

La homofobia internalizada parece afectar cada vez más la salud mental en adolescentes y jóvenes con orientación sexual diversa, por el conflicto interno que representa lo que debería ser y lo que en realidad es, que genera un rechazo hacía sí mismo y hacía otras personas que compartan la misma identidad sexual, debido a que 
la sociedad les recuerda a los individuos de la diversidad sexual, que tienen que ocultar su orientación (Borja \& Núñez, 2015; Pereira \& Rodrígues, 2015). Esta situación los lleva a vivir bajo la sombra de su propia identidad sexual, a realizar actividades en la clandestinidad de la sociedad para evitar ser juzgados y señalados, algunas personas homosexuales tienen una doble vida, una donde realizan las actividades que la sociedad señala y otra en donde son acorde a su identidad sexual.

Como se ha mencionado la homofobia internalizada se relaciona con problemas de salud mental tales como estrés, ansiedad, depresión e ideaciones suicidas, consumo de sustancias psicoactivas y alcohol (Gibbs \& Goldbach, 2015; Pineda Roa, 2013), que incrementa el riesgo de una muerte prematura. Moral, Valle, y García, (2013) realizaron una investigación con estudiantes universitarios de ciencias de la salud y demostraron que el $17 \%$ de los participantes manifestaron homofobia internalizada, de la cual señalaron tener pensamientos y deseos homosexuales e identidad no heterosexual. Asimismo, Piña y Aguayo, (2015) encontraron que los jóvenes universitarios manifestaron rechazo hacia la homosexualidad, tanto explícita como implícita de manera sutil. Es por ello que en un sentido amplio la homofobia internalizada se refiere al rechazo de la homosexualidad y al temor a ser considerado y tratado como una persona homosexual con independencia de la propia orientación sexual (Herek, 2007; Herek, Gillis, \& Cogan, 2009).

Es fundamental conocer los niveles de homofobia internalizada en edades tempranas como es la adolescencia y la juventud, para conocer y profundizar en este fenómeno el cual ha sido abordado en grupos de mayor edad, sin embargo los adolescentes y jóvenes debido a su etapa de desarrollo se considera una población vulnerable, así mismo hasta donde se indagó no se encontraron estudios de homofobia internalizada en etapa de la adolescencia, lo que sugiere que el fenómeno requiere ser profundizado en hombres como en mujeres, dado que en México la sociedad aún tiene roles de género muy marcados, normas culturales conservadoras. Por lo anterior, el objetivo general del estudio fue determinar la asociación entre el sexo y la edad en la homofobia internalizada en los adolescentes y jóvenes.
Y los objetivos específicos fueron:

- Describir la homofobia internalizada en los adolescentes y jóvenes.

- Comparar por sexo la homofobia internalizada en los adolescentes y jóvenes.

- Establecer la relación que existe entre la edad y la homofobia internalizada en los adolescentes y jóvenes.

\section{MÉTODO}

Se realizó un estudio descriptivo correlacional de corte transversal (Burns \& Grove, 2015) en 246 adolescentes y jóvenes de dos instituciones públicas con nivel socioeconómico bajo, procedentes de familias de área rural. El muestreo fue aleatorio simple con un poder estadístico de $90 \%$. Se incluyeron a los adolescentes y jóvenes que tuvieran entre 14 a 24 años de edad, debido a que la OMS (1986) contempla a la adolescencia y le juventud en esos rangos de edades. Se eliminaron a los participantes que no llenaron el instrumento en un $50 \%$.

\section{Instrumentos}

Se utilizó una cédula de datos personales que incluyó la edad y el sexo de los participantes. Edad se refiere a los años cumplidos de los adolescentes y jóvenes al día de la aplicación del instrumento y sexo a las características biológicas que diferencia al hombre y la mujer.

Para medir la homofobia internalizada se aplicó la Escala de Homonegatividad Internalizada (HNI-16) de Moral \& Valle, (2013), la cual esta diseñada para aplicarse a hombres y mujeres independientemente de una orientación o identidad sexual definida. La escala está integrada por 16 ítems tipo Likert con 5 opciones de respuesta (de 1 "totalmente en desacuerdo" a 5 "totalmente de acuerdo"). La puntuación total de la escala se consigue tras sumar los 16 ítems después de haber realizado la inversión de los 5 ítems correspondientes (ítems 1, 2, 3, 4 y 11). Las puntuaciones oscilan de 16 a 80, donde mayor puntuación representa mayor nivel de homofobia internalizada. El instrumento cuenta con un Alpha de Cronbach de .88. 


\section{Procedimiento}

Para la colecta de los datos, se solicitó el permiso de las autoridades de dos instituciones educativas, una universidad pública y una secundaria rural al norte del estado de Sinaloa, considerando que los participantes de ambas instituciones comparten escenarios similares con estrato socio-económico bajo, en una cultura prevaleciente de machismo y de religión católica.

Cuando se obtuvo la autorización de los directivos, se aleatorizaron las listas de los grupos. En el caso de los adolescentes que eran menores de edad se les otorgó previamente el consentimiento y asentimiento informado para que fuera firmado por sus padres y por los menores de edad y en el caso de los mayores de edad solo se les entregó el consentimiento informado el día de la aplicación del instrumento. Se asistió a los grupos seleccionados para aplicar el instrumento a todos aquellos que aceptaron participar en estudio. Antes de iniciar con la aplicación del instrumento, se les pidió a los participantes que respondieran lo más cercano a su realidad y se les explicó que sus respuestas eran completamente anónimas y confidenciales, además que los datos que se obtuvieran serían utilizados exclusivamente para fines de investigación. La aplicación del instrumento fue a lápiz y papel con una duración de aproximadamente 10 minutos. Al finalizar el llenado de los instrumentos se les agradeció su participación.

\section{Análisis de datos}

Los datos recolectados se analizaron mediante el Statistical Package for the Social Sciences (SPSS) en su versión 22 para Windows. Se realizó un análisis descriptivo para identificar frecuencias, porcentajes y medias para obtener la distribución de los 16 ítems del instrumento de Homonegatividad Internalizada y se analizó la confiabilidad del instrumento. Se aplicó un análisis de prueba estadística de la $U$ de Mann-Whitney para obtener la diferencia entre la variable del sexo en la homofobia internalizada. Se realizó una correlación de Spearman para conocer la relación entre la edad y la homofobia internalizada y se analizó un Modelo de Regresión Lineal Simple para conocer la asociación del sexo, la edad y la homofobia internalizada.

\section{Consideraciones éticas}

Esta investigación se apegó a las reglas presentadas en el reglamento de la Ley General de Salud en Materia de Investigación para la Salud (1983). A los adolescentes se les otorgó asentimiento y consentimiento informado y a los jóvenes mayores de edad se les otorgó solamente consentimiento informado para su participación en el presente estudio.

\section{RESULTADOS}

De acuerdo a las características de la muestra, se encontró que la participación fue ligeramente mayor en las mujeres con un $57.7 \%$ y un $42.3 \%$ en hombres ( $M=$ 1.58, $D E=$.49). Asimismo, la edad mínima de los participantes fue de 14 y 24 la edad máxima $(M=16.64$, $D E=2.72)$. La escala de Homonegatividad Internalizada (HNI-16), mostró un Alpha de Cronbach de .82 y una media del índice general de 40.39 ( $D E=14.80)$, lo que representa en general una manifestación media de homofobia internalizada. En la Tabla 1 se presenta la distribución de cada uno de los ítems de la escala.

De acuerdo a la distribución de las frecuencias en cada ítem de la escala de Homonegatividad Internalizada (HNI-16, ver Tabla 1), se puede observar que los ítems que presentan mayor homofobia internalizada son el 12 ( $E /$ hacer o responder a un coqueteo de tipo homosexual sería muy difícil para mí), el 15 (Yo buscaría ayuda profesional si llegará a darme cuenta que tengo sentimientos homoeróticos) y el 16 (Yo me sentiría contrariado, fuera de mí mismo, debido a la presencia de sentimientos homosexuales en $\mathrm{mi}$ ).

Para conocer la diferencia entre la homofobia internalizada por sexo, se realizó un análisis con la prueba estadística de la U de Mann-Whitney (ver Tabla 2), cuyos resultados indicaron que los hombres ( $M d n=44.53$, $D E=15.15)$ son los que manifiestan mayores niveles de homofobia internalizada en comparación con las mujeres $(M d n=367.50, D E=13.59)$. Las diferencias entre el sexo fueron significativas $(U=5,077.00, p<.001)$. 
Tabla 1. Distribución de los 16 ítems del HNI-16

\begin{tabular}{|c|c|c|c|c|c|c|c|c|c|c|c|}
\hline \multirow[t]{2}{*}{ Ítems } & \multicolumn{2}{|c|}{$\begin{array}{c}1 \\
C D\end{array}$} & \multicolumn{2}{|c|}{$\begin{array}{c}2 \\
E D\end{array}$} & \multicolumn{2}{|l|}{$\begin{array}{l}3 \\
1\end{array}$} & \multicolumn{2}{|l|}{$\begin{array}{c}4 \\
\mathrm{DA}\end{array}$} & $\begin{array}{c}5 \\
\text { DDA }\end{array}$ & \multicolumn{2}{|c|}{$M$} \\
\hline & $f$ & $\%$ & $f$ & $\%$ & $f$ & $\%$ & $F$ & $\%$ & $f$ & $\%$ & \\
\hline $\begin{array}{l}\text { 1. No me incomodaría si otras } \\
\text { personas supieran que tengo } \\
\text { sentimientos homosexuales. }\end{array}$ & 52 & 21.1 & 23 & 9.3 & 42 & 17.1 & 72 & 29.3 & 57 & 23.2 & 3.24 \\
\hline $\begin{array}{l}\text { 2. Me siento cómodo al hablar sobre } \\
\text { homosexualidad en situaciones } \\
\text { públicas. }\end{array}$ & 41 & 16.7 & 43 & 17.5 & 37 & 15.0 & 62 & 25.2 & 63 & 25.6 & 3.26 \\
\hline $\begin{array}{l}\text { 3. Siendo homosexual, yo no } \\
\text { cambiaría mi orientación sexual, } \\
\text { aunque pudiera hacerlo. }\end{array}$ & 37 & 15.2 & 47 & 19.3 & 49 & 20.2 & 56 & 23.0 & 54 & 22.2 & 3.18 \\
\hline $\begin{array}{l}\text { 4. No me incomoda el ser visto en } \\
\text { público con una persona obviamente } \\
\text { homosexual. }\end{array}$ & 41 & 16.7 & 27 & 11.0 & 31 & 12.6 & 73 & 29.7 & 74 & 30.1 & 3.46 \\
\hline $\begin{array}{l}\text { 5. La mayoría de los hombres } \\
\text { homosexuales no pueden mantener } \\
\text { una relación sentimental a largo } \\
\text { plazo. }\end{array}$ & 66 & 27.0 & 75 & 30.7 & 51 & 20.9 & 37 & 15.2 & 15 & 6.1 & 2.43 \\
\hline $\begin{array}{l}\text { 6. La mayoría de los hombres } \\
\text { homosexuales prefieren tener } \\
\text { encuentros sexuales anónimos. }\end{array}$ & 38 & 15.6 & 54 & 22.2 & 84 & 34.6 & 53 & 21.8 & 14 & 5.8 & 2.80 \\
\hline $\begin{array}{l}\text { 7. Los hombres homosexuales } \\
\text { tienden a mostrar su sexualidad } \\
\text { inapropiadamente. }\end{array}$ & 41 & 16.8 & 61 & 25.0 & 72 & 29.5 & 61 & 25.0 & 9 & 3.7 & 2.74 \\
\hline $\begin{array}{l}\text { 8. Los hombres homosexuales son } \\
\text { más promiscuos que los } \\
\text { heterosexuales. }\end{array}$ & 49 & 20.2 & 58 & 23.9 & 65 & 26.7 & 53 & 21.8 & 18 & 7.4 & 2.72 \\
\hline $\begin{array}{l}\text { 9. A menudo me siento intimidado al } \\
\text { andar en lugares de ambiente } \\
\text { homosexual. }\end{array}$ & 70 & 29.2 & 69 & 28.8 & 57 & 23.8 & 31 & 12.9 & 13 & 5.4 & 2.37 \\
\hline $\begin{array}{l}\text { 10. Las situaciones sociales con } \\
\text { hombres homosexuales me hace } \\
\text { sentir incómodo. }\end{array}$ & 71 & 29.6 & 81 & 33.8 & 57 & 23.8 & 18 & 7.5 & 13 & 5.4 & 2.25 \\
\hline $\begin{array}{l}\text { 11. No me incomoda el estar en bares } \\
\text { de ambiente homosexual. }\end{array}$ & 51 & 21.3 & 52 & 21.7 & 52 & 21.7 & 59 & 24.6 & 26 & 10.8 & 2.82 \\
\hline $\begin{array}{l}\text { 12. El hacer o responder a un } \\
\text { coqueteo de tipo homosexual sería } \\
\text { muy difícil para mí. }\end{array}$ & 37 & 15.7 & 40 & 17.0 & 56 & 23.8 & 56 & 23.8 & 46 & 19.6 & 3.14 \\
\hline $\begin{array}{l}\text { 13. El ser homosexual representaría } \\
\text { una desventaja para mí. }\end{array}$ & 71 & 29.7 & 69 & 28.9 & 55 & 23.0 & 31 & 13.0 & 13 & 5.4 & 2.36 \\
\hline $\begin{array}{l}\text { 14. Siento que lo mejor es evitar la } \\
\text { interacción personal o social con } \\
\text { personas homosexuales. }\end{array}$ & 84 & 35.0 & 82 & 34.2 & 45 & 18.8 & 20 & 8.3 & 9 & 3.8 & 2.12 \\
\hline $\begin{array}{l}\text { 15. Yo buscaría ayuda profesional si } \\
\text { llegara a darme cuenta que tengo } \\
\text { sentimientos homoeróticos. }\end{array}$ & 51 & 21.2 & 47 & 19.5 & 50 & 20.7 & 66 & 27.4 & 27 & 11.2 & 2.88 \\
\hline $\begin{array}{l}\text { 16. Yo me sentiría contrariado, fuera } \\
\text { de mí mismo, debido a la presencia } \\
\text { de sentimientos homosexuales en mí. }\end{array}$ & 50 & 20.8 & 63 & 26.3 & 59 & 24.6 & 42 & 17.5 & 26 & 10.8 & 2.71 \\
\hline
\end{tabular}

Nota: $\mathrm{CD}=$ Completamente en desacuerdo, $\mathrm{ED}=$ En desacuerdo, I= Indiferente, $\mathrm{DA}=\mathrm{De}$ acuerdo, DDA= Definitivamente de acuerdo; $\mathrm{M}=\mathrm{Media}$ 
Tabla 2. Diferencia por sexo en Homofobia Internalizada

\begin{tabular}{llllllll}
\hline & Sexo & $n$ & Media & Mdn & $D E$ & $U$ & $p$ \\
\hline HI & Hombre & 104 & 45.10 & 44.53 & 15.15 & 5077.00 & $<.001$ \\
& Mujer & 142 & 36.94 & 37.50 & 13.59 & & \\
\hline
\end{tabular}

Nota: $\mathrm{HI}=$ Homofobia Internalizada, $\mathrm{DE}=$ Desviación Estándar, U= U de Mann-Whitney, $\mathrm{n}=246, p<.001$

Respecto a la relación de la edad y la homofobia internalizada se llevó a cabo la prueba de correlación de Spearman. Los resultados demuestran que la edad se relaciona negativamente con la homofobia internalizada $(r=-.22, p<.001)$. Por lo que se puede señalar que los participantes de menor edad manifiestan mayores niveles de homofobia internalizada.

Para conocer la asociación entre el sexo y la edad en la homofobia internalizada se realizó un Modelo de Regresión Simple y los resultados se presentan en la Tabla 3. El modelo fue significativo al demostrar que el $11.2 \%$ de la variación en la homofobia internalizada se debió al sexo y la edad $\left(F_{[2,243]}=15.40, p<.001\right)$.

Tabla 3. Regresión lineal simple del sexo, la edad con la homofobia internalizada

\begin{tabular}{lcccc}
\hline Modelos & $B$ & $\begin{array}{c}\text { Error } \\
\text { Estándar }\end{array}$ & Beta & Valor de $p$ \\
\hline Sexo & -7.72 & 6.06 & -.26 & $<.001$ \\
Edad & -1.06 & 1.81 & -.20 & $<.001$ \\
\hline
\end{tabular}

Nota: $\mathrm{DE}=$ Desviación Estándar, $\mathrm{U}=\mathrm{U}$ de Mann-Whitney, $\mathrm{n}=246, \mathrm{p}<.001$

\section{DISCUSIÓN}

El presente estudio demostró que los participantes manifestaron homofobia internalizada. Por lo que se puede decir que los adolescentes y jóvenes rechazan su orientación sexual y pudiera deberse a los roles de género. Los roles de género son los ideales de una cultura de lo que debe ser el hombre o la mujer, así como, las cualidades, los deberes y las expectativas de género de acuerdo a la cultura en la que el individuo se desarrolla (Nagoshi, Adams, Terrell, Hill, Brzuzy, \& Nagoshi, 2008).

Butler (2007) señala que "todo lo que somos es una imitación, una sombra de la realidad". Por lo cual señala que el género es el resultado de moderar las diferencias de género de forma coercitiva. A su vez, señala que la heterosexualidad se acuñe a las normas de género, es por ello que la homofobia actúa sobre la homosexualidad como un género no reconocido o fallido. Lo anterior designa a los hombres gay como "afeminados" "maricones", a las lesbianas como "masculinas" "marimachas" y a los transexuales como "depravados" "enfermos". Lo anterior impacta en la manifestación de la homofobia internalizada, debido a que la persona puede perder su identidad de género y no considerarse como un "hombre" o una "mujer". Por lo que Butler (2007) menciona que no hay género propio del hombre y de la mujer, indica que el género se deriva de un sistema dominador que se apropia de la cultura de los sexos. A su vez, Preciado (2002) señala que el género "son trozos de lenguaje cargados históricamente del poder de conceder un cuerpo como masculino o como femenino, así como de sancionar los cuerpos que amenazan la coherencia del sistema sexo/género hasta el punto de someterlos a procesos quirúrgicos de cosmética sexual".

Asimismo, el presente estudio encontró que los hombres manifestaron mayores niveles de homofobia internalizada en comparación con las mujeres. Lo anterior puede deberse a los ideales sociales heteronormativos de la virilidad masculina, con la finalidad de mantener el dominio sobre la mujer. Norton (1997) señala que para mantener los ideales tradiciones de la superioridad masculina, debe adherirse a los ideales tradicionales culturales del comportamiento masculino y a la orientación heterosexual. Por lo cual, los hombres que tienen creencias sobre los roles de género, la identidad de género, la orientación sexual y el uso de violencia y agresión para mantener el poder, están impulsados por ideologías que comparten en común. Nascimento, (2014) señala que una identidad masculina se asocia con la homofobia, esto puede explicarse debido a que los hombres consideran que deben mantener las reglas sociales de la masculinidad, principalmente en el contexto Mexicano, que prevalece el machismo y las creencias de una imagen masculina aparente, muestre mayor hombría, lo que sería incongruente al ser homosexual. Preciado (2002) menciona que los hombres y las mujeres son construcciones metonímicas del sistema heterosexual de producción y de reproducción, que somete a las mujeres que conforma la obligación en la reproducción y en el trabajo sexual, sin importar su interés en el erotismo y placer sexual y al hombre ser considerado como el centro mecánico de la producción del impulso sexual. 
A su vez, el resultado que se obtuvo en este estudio en relación a la diferencia entre los niveles de homofobia internalizada en hombres y mujeres, concuerda con el estudio de España, Guerrero, Farré, Canella-Soler, y Abós, (2001), en donde señalan que las mujeres parecen ser más permisivas en cuanto a la homosexualidad debido a aspectos culturales antiguos que señalan al hombre como el responsable de "preservar" el rol de género, las instituciones y las creencias tradicionales, permitiéndole así, a la mujer, tomar actitudes más "tolerantes". En cambio los hombres conservan mayores ideologías machistas y homofóbicas, que impide incluso la demostración de afecto hacia otros hombres principalmente, independientemente incluso de su identidad u orientación sexual (Nascimento, 2014). En el estudio llevado a cabo por Baena, Calvente, y Díaz, (2013) también se demostró que los hombres presentaban mayores niveles de homofobia que las mujeres y este mismo resultado se encontró en el estudio de García (2015), demostrando que las mujeres tienen actitudes más favorables frente a la homosexualidad. También, en el estudio de Carrera-Fernández, Lameiras-Fernández, Rodríguez-Castro, y Vallejo-Medina, (2013) los hombres tuvieron una puntuación más alta que las mujeres en cuanto a actitudes homófobas y bullying, lo cual indicaría que así como los hombres tienden a presentar mayor homofobia internalizada o externalizada, también parecen ser más propensos a tener actitudes de intimidación y acoso frente a las personas de diferente orientación sexual. Actualmente, el bullying homofóbico tanto verbal como psicológico está muy presente en los jóvenes pertenecientes a la comunidad LGBT (Blais, Gervais, \& Hébert, 2014).

Estos eventos de homofobia y bullying en contra de jóvenes con una identidad sexual diferente a la heterosexual, les pueden traer problemas en diversas áreas, sobre todo, en su salud mental. No es la orientación sexual en sí, sino la discriminación y estigmatización lo que puede generar estrés y malestar a lo largo de la vida, con sus consecuentes efectos en la salud mental (Carro, 2015). Espada, Morales, Orgilés, y Ballester (2012) en su estudio, demuestran que los adolescentes no heterosexuales muestran un mayor riesgo de padecer depresión con respecto a sus compañeros heterosexuales, mientras que Fernández y Vázquez, (2013) realizaron un estudio en jóvenes donde encontraron que 1 de cada 3 con una identidad no heterosexual tuvieron pensamientos suicidas y a su vez de los jóvenes que tuvieron esos pensamientos más de la mitad de ellos llevaron a cabo el intento de suicidio.

Otro de los resultados encontrados en este estudio fue la relación negativa entre edad y homofobia internalizada, lo que nos indica que, a mayor edad, hay menor presencia de homofobia internalizada. Este resultado concuerda con García (2015) que señala que los adolescentes más jóvenes (14 - 15 años) muestran un mayor nivel de homofobia que los de mayor edad (17 18 años). Mientras que en el estudio de Borja y Núñez (2015) demuestra que los individuos más jóvenes tienden a mostrar mayor rechazo hacia el colectivo homosexual. Los autores proponen que algunas de las razones involucradas en este cambio del pensamiento del adolescente a medida que va creciendo, tendrían que ver con la madurez que va adquiriendo a través de los años, así como la influencia de asignaturas escolares que tengan contenidos sobre la igualdad y tolerancia. Asimismo, Poteat y Anderson (2012) demostraron que los adolescentes más jóvenes muestran mayores niveles de homofobia y prejuicio, que se va reduciendo conforme a la edad avanza.

También se encontró que el sexo y la edad son determinantes que contribuyen a la manifestación de homofobia internalizada. Por lo que se puede decir que los hombres que son de menor edad tienen mayor probabilidad de manifestar homofobia internalizada. Lo anterior es similar a lo reportado por McLaren (2015), donde demostró en su estudio que los participantes de menor edad se relacionaron con mayor presencia de homofobia internalizada, sin embargo, no se encontró asociación significativa con el sexo. Lo anterior pudiera deberse a que el estudio fue realizado en un contexto sociocultural diverso al del presente estudio. Una investigación realizada en México, demostró que los hombres indicaban mayor presencia de homofobia internalizada en comparación con las mujeres, esto puede atribuirse al contexto cultural, a través de la construcción social del género y conservadora, debido a que en el contexto mexicano los hombres con una identidad no heterosexual son más señalados o violentados que las mujeres, al presentar una imagen feminizada, lo cual puede asociarse con una opresión internalizada (Campo-Arias \& Herazo, 2008; Ortíz, 2005). Asimismo, Díaz-Loving, et al (2015) señala que el ser hombre o mujer está mediado por los ideales culturales tradicionales en la que el individuo se desarrolla, así 
como también, las creencias y normales que rigen la cultura para la determinación del comportamiento de los individuos.

Asimismo, se puede señalar que la construcción de la homosexualidad se presenta desde lo externo hacia lo interno, esto quiere decir que primeramente se dan los actos o acciones sexuales y posteriormente la consciencia homosexual. Castañeda (2000) señala que la construcción homosexual es más frecuente en los hombres que en las mujeres, debido a que los hombres por lo general se inician en los actos sexuales y las mujeres regularmente requieren de sentimientos para su iniciación. A su vez Castañeda (2000) señala que de acuerdo a estudios previos los hombres toman conciencia de sus deseos homosexuales a más temprana edad en comparación con las mujeres. Los hombres comienzan a crear conciencia de sus deseos homosexuales aproximadamente a los 13 años, a los 15 años tiende a presentarse su primera experiencia homosexual, a los 22 años tienen su primera relación amorosa y a los 28 años en promedio adquieren una identidad gay positiva. Sin embargo, las mujeres crean conciencia un poco después, a los 14 años toman conciencia de sus deseos homosexuales, a los 20 años en promedio tienen su primera experiencia homosexual, a los 23 años su primera relación amorosa y aproximadamente a los 30 años adquiere su identidad lésbica positiva.

\section{Conclusiones}

Con lo anterior, se puede concluir que son los hombres quiénes presentan mayores niveles de homofobia internalizada y que los adolescentes en etapa temprana son los que manifiestan mayores niveles de homofobia internalizada, debido a los patrones culturales y sociales en los cuales presiden.

El sexo y la edad son factores importantes para el diseño de intervenciones enfocadas a la prevención de la violencia, discriminación y bullying homofóbico con elementos culturales pertinentes al contexto de los adolescentes y jóvenes, tales como el machismo, el familismo y marianismo, esos aspectos culturales que afectan de manera negativa convertirlos en elementos positivos que permitan fortalecer la autoestima y la mejora de la toma de decisiones asertivas en los adolescentes y jóvenes. En el contexto escolar, es importante incluir cursos o asignaturas enfocadas a la sexualidad que aborden a la diversidad sexual e incluyan aspectos asociados a las actitudes positivas a la población de las minorías sexuales y que permitan integrar con equidad y evitar rechazo social por una orientación o identidad sexual diversa.

En los centros comunitarios y en los sistemas de salud, especialmente, en las áreas de planificación familiar y atención a la salud mental, es de relevancia incluir acciones enfocadas la detección de malestares de tristeza, conductas de riesgo, síntomas depresivos, que puedan derivarse de la manifestación de homofobia internalizada y así evitar riesgos de salud mental en la etapa de la adolescencia o juventud.

De acuerdo a la revisión de la literatura y hasta donde se indagó, son pocos los estudios que se han realizado en poblaciones heterosexuales $y$ en adolescentes en etapa temprana relacionada con aspectos de orientación sexual y homofobia internalizada. Por lo anterior, se recomienda realizar investigaciones con enfoque mixto, debido a que a través de esa metodología permitiría conocer el aporte cualitativo de las experiencias, sentimientos y contextos sociales y culturales que tienen los adolescentes y jóvenes en relación a la aceptación o rechazo de una orientación no heterosexual y el aporte cuantitativo de los potenciales factores de riesgo y protección que se asocien a la homofobia internalizada con la finalidad de explicar más ampliamente el fenómeno de estudio y generar nuevas propuestas de intervenciones para la disminución de riesgos en la salud mental y física de los adolescentes y jóvenes.

A su vez, a través del desarrollo de proyectos de investigación, se propone generar acciones sociales y políticas públicas enfocadas al reconocimiento $y$ aceptación de la diversidad sexual, con la finalidad de disminuir actitudes homofóbicas e ideologías heterosexistas, de tal forma que a través de las políticas formuladas reduzca los índices de violencia de género y la discriminación y aumente la equidad de género y permita la inclusión social de la diversidad sexual en un ambiente positivo libre de violencia y por ende, la reducción de malestar psicológico, como lo es el estrés, ansiedad y síntomas depresivos. 


\section{Limitaciones}

Los datos fueron colectados en un municipio del norte de Sinaloa, por lo que la generalización de los resultados debe realizar en poblaciones con características similares. Otra de las limitaciones del estudio fue que se consideró una muestra pequeña, por lo que se recomienda hacerlo en muestras más representativas e incluir estudiantes de preparatoria. Para futuros estudios se recomienda incorporar más variables que pudieran estar prediciendo la manifestación de homofobia internalizada en adolescentes y jóvenes. El estudio fue de tipo transversal, lo que limita una evaluación más precisa y no permite conocer la causalidad. Las limitaciones anteriormente señaladas son de importancia para atender en próximas investigaciones.

\section{REFERENCIAS}

Baena, P. D., Calvente, P. M. del R., \& Díaz, S. M. (2013). Homosexualidad, ¿Qué actitud tienen los universitarios al respecto? Revista Electrónica de Investigación Docencia Creativa, 2, pp. 130-137.

Barrientos, J., \& Cárdenas, M. (2013). Homofobia y calidad de vida de gay y lesbianas: Una mirada psicosocial. Psykhe (Santiago), 22(1), pp. 314. DOI: $10.7764 /$ psykhe.2013.22.10

Blais, M., Gervais, J., \& Hébert, M. (2014). Internalized homophobia as a partial mediator between homophobic bullying and self-esteem among youths of sexual minorities in Quebec (Canada). Ciência \& Saúde Coletiva, 19(3), pp. 727-735. DOI: 10.1590/141381232014193.16082013

Borja, G. J., \& Núñez, D. T. (2015). Actitudes homófobas en adolescentes. Reflexiones para el cambio. Revista Estudios Y Cultura, 72, pp. 75-87.

Burns, N., \& Grove, S. K. (2015). The Practice of Nursing Research: Conduct, critique, and utilization. (Elsevier/Saunders, Ed.) (5th ed.). St. Louis, Missouri.

Butler, J. (2007). El género en disputa. El feminismo y la subeversión de la identidad. Barcelona: Paidós.

Campo-Arias, A., \& Herazo, E. (2008). Homofobia en estudiantes de medicina: una revisión de los diez últimos años. Revista MedUNAB, 11(2), pp. 120-123.

Caricote, Á. E. A. (2009). La salud sexual en la adolescencia tardía. Educere, 13(45), pp. 415-425.

Carrera-Fernández, M.-V., Lameiras-Fernández, M., Rodríguez-Castro, Y., \& Vallejo-Medina, P. (2013). Bullying Among Spanish Secondary Education Students: The Role of Gender Traits, Sexism, and Homophobia. Journal of Interpersonal Violence, 28(14), pp. 29152940. DOI: $10.1177 / 0886260513488695$
Carro, S. C. (2015). Efectos de la homonegatividad en la salud mental de mujeres lesbianas. (Tesis). Montevideo, Uruguay: Facultad de Psicología, Universidad de la República. Recuperada de: https://www.colibri.udelar.edu.uy/jspui/bitstream/123456789/579 3/1/Carro\%2c\%20Camila.pdf

Castañeda, M. (2000). La experiencia homosexual. México: Paidós.

Díaz-Loving, R., Saldívar, A., Armenta-Hurtarte, C., Reyes, N., López, F., Moreno, M.,... Correa, F. (2015). Creencias y Normas en México: Una Actualización del Estudio de las Premisas Psico-Socio-Culturales. Psykhe, 24(2), pp. 1-25. DOI:10.7764/psykhe.24.2.880

Encuesta Nacional de Salud y Nutrición. (2012). Encuesta Nacional de Salud y Nutrición 2012. Resultados por entidad federativa. Sinaloa. Recuperado el 27 de Junio del 2016 de http://ensanut.insp.mx/informes/Sinaloa-OCT.pdf

Espada, J. P., Morales, A., Orgilés, M., \& Ballester, R. (2012). Autoconcepto, ansiedad social y sintomatología depresiva en adolescentes españoles según su orientación sexual. Ansiedad Y Estrés, 18(1), pp. $31-41$.

España, A. A., Guerrero, R. A., Farré, J. M., Canella-Soler, J., \& Abós, R. (2001). La homofobia en el medio universitario. Un estudio empírico. Cuadernos de Medicina psicosomática y Psiquiatria de Enlace, 57, pp. 41-55.

Fernández, R. M. del C., \& Vázquez, C. F. (2013). En torno al rechazo, la salud mental y la resiliencia en un grupo de jóvenes universitarios gays, lesbianas y bisexuales. Revista Griot, 6(1), pp. 44-65.

García, B. M. (2015). Orientación sexual y homofobia en adolescentes españoles. "Tesis para optar el título de Licenciatura en psicología". Universitat Jaume I. Ciudad de Castellón de la Plana, España.

Gibbs, J. J., \& Goldbach, J. (2015). Religious Conflict, Sexual Identity, and Suicidal Behaviors among LGBT Young Adults. Archives of Suicide Research, 19(4), pp. 472-488. DOI: 10.1080/13811118.2015.1004476

Herek, G. M. (2004). Beyond "Homophobia": Thinking about sexual prejudice and stigma in the twenty-first century. Sexuality Research and Social Policy, 1(2), pp. 6-24. DOI: 10.1525/srsp.2004.1.2.6

Kaysen, D. L., Kulesza, M., Balsam, K. F., Rhew, I. C., Blayney, J. A., Lehavot, K., \& Hughes, T. L. (2014). Coping as a mediator of internalized homophobia and psychological distress among young adult sexual minority women. Psychology of Sexual Orientation and Gender Diversity, 1(3), pp. 225-233. DOI: 10.1037/sgd0000045

Lozano, V. I., \& Díaz-Loving, R. (2010). Medición de la homofobia en México: Desarrollo y validación. Revista Iberoamericana de Diagnóstico Y Evaluación Psicológica, 2(30), pp. 105-124.

Lozano, V. I., \& Rocha, S. T. E. (2011). La homofobia y su relación con la masculinidad hegemónica en México. Revista Puertorriqueña de Psicología, 22, pp. 101-121.

McLaren, S. (2015). Gender, Age, and Place of Residence as Moderators of the Internalized Homophobia Depressive Symptoms Relation Among Australian Gay Men and Lesbians. Journal of Homosexuality, 62(4), pp. 463-80. DOI: 10.1080/00918369.2014.983376 
Moral, D. la R. J., \& Valle, D. la O. A. (2013). Dimensionalidad, Consistencia Interna y Distribución de la Escala Homonegatividad Internalizada en Estudiantes Mexicanos de Ciencias de la Salud. Acta de Investigación Psicológica, 3(1), pp. 986-1004.

Moral, D. la R. J., Valle, D. la O. A., \& García, C. C. H. (2013). Modelos Predictivos de Homonegatividad Internalizada en Estudiantes de Ciencias de la Salud. Acta de Investigación Psicológica, 3(3), pp. 12481263.

Nagoshi, J. L., Adams, K. A., Terrell, H. K., Hill, E. D., Brzuzy, S., \& Nagoshi, C. T. (2008). Gender differences in correlates of homophobia and transphobia. Sex Roles, 59(7-8), pp. 521-531. DOI: 10.1007/s11199008-9458-7.

Nascimento, M. (2014). Hombres, masculinidades y homofobia: Apuntes para la reflexión desde lo conceptual y de lo político. Conexões PSI, 2(2), pp. 41-59.

Newcomb, M., \& Mustanski, B. (2010). Internalized homophobia and internalizing mental health problems: a meta-analytic review. Clinical Psychology Review, 30(8), pp. 1019-29. DOI: 10.1016/j.cpr.2010.07.003.

Norton, J. (1997). "Brain says you're a girl, but I think you're a sissy boy": Cultural origins of transphobia. Journal of Gay, Lesbian, and Bisexual Identity, 2, pp. 139-164. DOI: 10.1023/A:1026320611878.

Ortiz, H. L. (2005). Influencia de la opresión internalizada sobre la salud mental de bisexuales, lesbianas y homosexuales de la Ciudad de México. Salud Mental, 28(4), pp. 49-65

Peixoto, C. J. M., Fonseca, L., Almeida, S., \& Almeida, L. (2012). Escuela Y Diversidad Sexual - ¿Que Realidad? Educação Em Revista, 28(3), pp. 143-158.

Pereira, H., \& Rodrígues, P. (2015). Internalized Homophobia and Suicidal Ideation among LGB Youth. Journal of Psychiatry, 18(2), pp. 14-168. DOI: 10.4172/Psychiatry.1000229

Pineda Roa, C. A. (2013). Factores asociados con riesgo de suicidio de adolescentes y jóvenes autoidentificados como lesbianas, gays y bisexuales: estado actual de la literatura. Revista Colombiana de Psiquiatría, 42(4), pp. 333-349. DOI: 10.1016/S0034-7450(13)70030-

Piña, O. J. M., \& Aguayo, R. H. B. (2015). Homofobia en estudiantes universitarios de México. Región Y Sociedad, 64, pp. 5-35.

Poteat, V. P., \& Anderson, C. J. (2012). Developmental changes in sexual prejudice from early to late adolescence: The effects of gender, race, and ideology on different patterns of change. Developmental Psychology, 48(5), pp. 1403-1415. DOI: 10.1037/a0026906

Preciado, B. (2002). Manifiesto contra-sexual. España: Opera Prima.

Secretaría de Salud. (1983). REGLAMENTO de la Ley General de Salud en Materia de Investigación para la Salud. Recuperado el 17 de Julio del 2016 de: http://www.salud.gob.mx/unidades/cdi/nom/compi/rlgsmis.html

Secretaría de Salud. (2014). Depresión y Suicidio en México. Recuperado el 27 de Junio del 2016 de http://www.spps.gob.mx/avisos/869depresion-y-suicidio-mexico.html
Silva-Escorcia, I., \& Mejía-Pérez, O. (2015). Autoestima, adolescencia y pedagogía. Revista Electrónica Educare, 19(1), pp. 241-256. DOI: 10.15359/ree.19-1.13

Teixeira-Filho, F. S., Rondini, C. A., \& Bessa, J. C. (2011). Reflexões sobre homofobia e educação em escolas do interior paulista. Educação $E$ Pesquisa, 37(4), pp. 725-741. DOI: 10.1590/S151797022011000400004

World Health Organization. (1986). La salud de los jóvenes: un desafío para la sociedad. Recuperado el 17 de Julio del 2016 de: http://apps.who.int/iris/bitstream/10665/36922/1/WHO_TRS_731_ spa.pdf

World Health Organization. (2016). La depresión. Recuperado el 27 de Junio del 2016 de http://www.who.int/mediacentre/factsheets/fs369/es/

Xu, W., Zheng, L., Xu, Y., \& Zheng, Y. (2017). Internalized homophobia, mental health, sexual behaviors, and outness of gay/bisexual men from Southwest China. International Journal for Equity Health, 16(36), pp. 1-10. DOI: 10.1186/s12939-017-0530-1 